\title{
The concept of 'Semi-clean colon' using the pit pattern classification system has the potential to be acceptable in combination with a $<3$-year surveillance colonoscopy
}

\author{
YASUHARU MAEDA, SHIN-EI KUDO, KUNIHIKO WAKAMURA, HIDEYUKI MIYACHI, \\ MASASHI MISAWA, YUICHI MORI, NORIYUKI OGATA, TOYOKI KUDO, KENTA KODAMA, \\ TOMOKAZU HISAYUKI, TAKEMASA HAYASHI, ATSUSHI KATAGIRI and FUMIO ISHIDA
}

Digestive Disease Center, Showa University Northern Yokohama Hospital, Yokohama, Kanagawa 224-8503, Japan

Received July 1, 2016; Accepted June 8, 2017

DOI: $10.3892 / 01.2017 .6491$

\begin{abstract}
Histological features of colorectal lesions are currently evaluated via a magnifying chromoendoscopy [pit pattern (PIT) classification]. Advanced histological features are rarely observed in diminutive $(\leq 5 \mathrm{~mm})$ adenomatous polyps (DAPs). The Japanese guidelines indicate that diminutive neoplastic lesions without carcinomatous findings may be left untreated and followed up. At the present institution, DAPs with type IIIL PIT are left untreated in various cases, whereas lesions with type III, IV or V PIT are typically resected via routine colonoscopy. This retrospective study aimed to assess the management of DAPs using PIT classification. The participants of the study included patients $<30$ years previously referred for an initial colonoscopy, then reobserved for $<3$ years following the procedure. Participants were classified into three groups: Group A, Patients with type IIIL PIT DAP left untreated (semi-clean colon group); group B, patients with all neoplastic polyps, including DAPs, resected (clean colon group); and group C, patients without any adenomatous polyps (internal control group). The cumulative incidence of the index lesions (ILs) at the follow-up colonoscopy was analysed among the three groups. A total of 4,313 patients were enrolled in the study, with categorization as follows: Group A, 1,246; group B, 1,205; and group C, 1,862 patients. ILs were detected in group A, 100 (8.0\%); group B, 104 (8.6\%); and group C, 29 $(1.6 \%)$ patients. There was no significant difference observed between groups A and B. It was verified that removing the type IIIL PIT DAPs did not decrease the incidence of ILs within a 3-year time period. Therefore, these polyps may be left untreated in combination with patient reobservation
\end{abstract}

Correspondence to: Dr Shin-Ei Kudo, Digestive Disease Center, Showa University Northern Yokohama Hospital, 35-1 Chigasaki-Chuo, Tsuzuki-ku, Yokohama, Kanagawa 224-8503, Japan

E-mail: kudos@med.showa-u.ac.jp

Key words: magnifying endoscopy, pit pattern, colorectal diminutive adenomatous polyp, advanced neoplasia at an appropriate time interval, potentially one equal to that suggested following a polypectomy.

\section{Introduction}

Colorectal cancer (CRC) is the third most common cause of death due to cancer in Japan. The adenoma-carcinoma sequence is thought to be the primary route involved in the development of CRC (1), and neoplastic lesions found during colonoscopy are associated with a future risk of neoplasms. It has been demonstrated that colonoscopy with polypectomy reduces the risk of recurrent $\mathrm{CRC}$ and mortality $(2,3)$; the colonoscopy surveillance interval is based on risk stratification according to the number of adenomas, the maximum size of polyps and the histopathological findings of all resected lesions $(4,5)$. This is the primary screening method used in the US and several European countries $(6,7)$. On the other hand, the most suitable colonoscopy interval was described briefly in the Japanese guidelines as follows: ' $<3$ years surveillance colonoscopy is recommended after polypectomy' (8). Additionally, Japanese guidelines indicate diminutive neoplastic lesions without carcinomatous findings can be left untreated and followed up (i.e., semi-clean colon). The vast majority of polyps removed during a colonoscopy are diminutive $(\leq 5 \mathrm{~mm}$ in size), and approximately half of the diminutive polyps are adenomas (9-11). Furthermore, only a small percentage of diminutive adenomatous polyps (DAPs) contain advanced histological features (12). Indeed, some endoscopists in Japan leave DAPs unresected after a detailed observation and a close follow-up. This is because povlypectomy may be unnecessary because of the low prevalence of advanced features in these polyps, and it may be unnecessary to expose patients to added risks during colonoscopy (13).

In the PIT classification system described by Kudo et al (14) (Fig. 1), type I and type II lesions are defined as having non-neoplastic patterns; type IIIL, type IIIs and type IV are adenomatous; and type VI and type VN are cancerous. Concerning type IIIL, a previous study reported that lesions in this category exhibited no invasive characteristics (15). However, type III is a standard pit pattern observed in depressed-types of early cancer, and type IV lesions often 
contain characteristics of advanced neoplasia (e.g., high-grade adenomas or villous components).

Magnifying a chromoendoscopy with PIT assessment increases the diagnostic accuracy for both distinguishing adenomas from hyperplastic polyps (85-96\%) as well as diagnosing massively invasive submucosal colorectal cancer which has the possibility of metastasising (76.4-89.3\%) (16-18). Moreover, this classification system has a good-to-excellent inter-observer agreement (0.78-0.96) and could be used to accurately diagnose patients before treatment $(19,20)$.

Recently, the number of patients taking antiplatelet or anticlotting medications to prevent cerebral vascular disease or myocardial infarction has increased. For patients who continue to take their antiplatelet or anticlotting medications, or those who have a large number of polyps with difficulty resecting at a time, we often recommend leaving type IIIL PIT DAPs untreated and conducting a follow-up without an immediate re-examination (semi-clean colon strategy). However, the risk and adequate surveillance intervals of the semi-clean colon are not clear.

Therefore, this retrospective case-control study was designed to investigate the feasibility of a semi-clean colon.

\section{Materials and methods}

Patients and study design. In this retrospective case-control study, we acquired data from a database of colonoscopy examinations that had been prospectively recorded in Showa University Northern Yokohama Hospital (Yokohama, Japan), a tertiary referral centre in Japan. The study subjects consisted of patients over 30 years of age who were referred for an initial total colonoscopy and were followed up for $>3$ years from April 2001 to March 2014.

Patients who met the following criteria were excluded from the study: Those that were not caecal intubation; those with lesions $>5 \mathrm{~mm}$ in size and/or classified as type IIIS, IV, or V PIT that were initially left untreated; those that had a detected number of adenomas polyps $\geq 10$; and those with a history of familial adenomatous polyposis, Lynch syndrome, advanced colorectal cancer, inflammatory bowel disease, or a colectomy. All participants provided written informed consent, and the study was conducted according to the Declaration of Helsinki.

Participants were classified into three groups according to histological findings and treatment at the time of the initial colonoscopy: Group A, patients with type IIIL DAPs that were left untreated (semi-clean colon group); group B, patients in whom all neoplastic polyps, including DAPs were resected (clean colon group); and group $\mathrm{C}$, patients without any adenomatous polyps (internal control group). Furthermore, groups A and B were classified as high- and low-risk patients, based on the number of adenomas, maximum size of the polyps and the histopathological findings according to the US guidelines as follows: Low-risk, 1-2 tubular adenomas $<10 \mathrm{~mm}$; high-risk, 3-10 adenomas or $\geq 1$ advanced adenoma (6).

We retrospectively reviewed the database and medical records. The study protocol was approved by the ethics committee of Showa University Northern Yokohama Hospital (no. 1,410-05) and registered in the UMIN clinical trial registry (UMIN000016367).
Endoscopic procedures. Prior to the examination, patients underwent a bowel preparation with 2-3 liters of a polyethylene glycol solution. Diazepam and butyl scopolamine were used intravenously for sedation and prevention of peristalsis. All patients underwent total colonoscopies with magnifying endoscopes (CF-240ZI, CF-H260AZI, PCF-240ZI; Olympus Corp., Tokyo, Japan) using approximately 80- to 100 -fold magnification. During the magnifying observation, on-site endoscopists first sprayed the target lesion with indigo carmine, and if necessary, they then stained the lesion with crystal violet. All detected lesions were diagnosed on the basis of the PIT classification system described by Kudo et al (14). Each diagnosis was recorded in the database just after the colonoscopy. Lesion size, location and shape [Paris classification 10 (21)] were also measured and recorded. The lesions diagnosed as non-neoplastic were left untreated. Conversely, all neoplastic lesions, except for type IIIL PIT DAPs, were completely removed. Type IIIL PIT DAPs were left untreated at the discretion of the on-site endoscopists as Fig. 2 .

Histopathological evaluation. All resected specimens were fixed in $10 \%$ formalin, embedded in paraffin, serially sectioned, and stained using haematoxylin and eosin. Experienced gastrointestinal pathologists evaluated all pathological specimens. Histopathological diagnoses were determined based on the World Health Organization criteria (22).

Outcome measures. As a primary outcome measure, the cumulative incidence of index lesions (ILs) for the follow-up colonoscopy was analysed among the three groups. The ILs diagnosed during the follow-up colonoscopy were defined as follows: Large adenomatous polyps $\geq 10 \mathrm{~mm}$, high-grade dysplasia (intramucosal cancer) and invasive cancers. We also evaluated the incidence of invasive and interval cancers. In this study, interval cancer was defined as invasive cancer diagnosed within the 36 months following a baseline colonoscopy. This definition has been used in multiple previous studies $(23,24)$.

We also compare the ILs incidence between untreated DAPs (group A) and resected DAPs group in group B.

Statistical analysis. For the statistical analyses, a computerised database was designed using R (v. 2.13.0; The R Foundation for Statistical Computing, Vienna, Austria). Quantitative data were expressed as the mean and standard deviation values. The cumulative incidence rates over the maximum follow-up period among the three groups were compared using the Grey test. Statistical significance was evaluated using the Chi-squared test and $\mathrm{P}<0.05$ was considered to indicate a statistically significant difference. The Bonferroni method was used to counteract the problem of multiple comparisons. With regard to multivariable analysis, logistic regression analysis was conducted and $\mathrm{P}<0.05$ was considered to indicate a statistically significant difference. All authors had access to the study data and reviewed and approved the final manuscript.

\section{Results}

Subjects and outlines of the initial colonoscopy. A total of 5,391 patients were analysed and 1,078 patients were 


\section{Non-neoplastic}
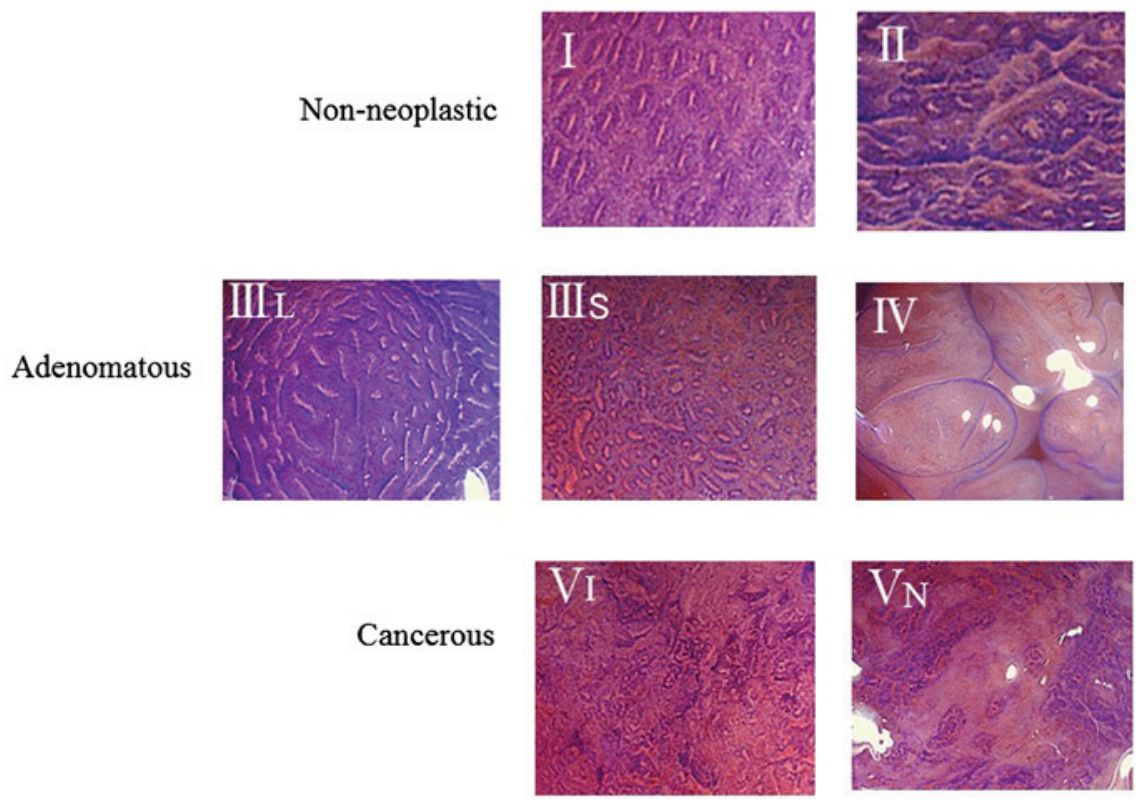

Figure 1. Kudo's pit pattern classification was composed seven type pit pattern. Type I and II indicate non-neoplastic lesions, type IIIL, IIIs and IV indicate adenomatous, and type VI and VN indicate cancerous.

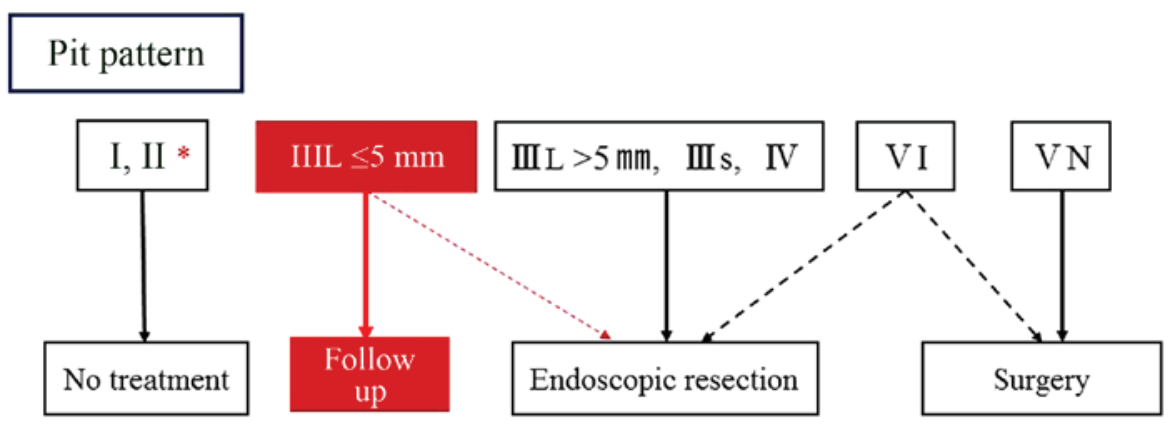

* Except for SSA/P and traditional serrated adenoma

Figure 2. In our management of colorectal lesions using pit pattern classification the lesions diagnosed as type I and II are not needed treatment except for the lesion suspected SSA/P and traditional serrated adenoma. The lesions diagnosed as type IIIL with no more than 5 mm in size are left untreated and followed up. The other lesions need treatment.

excluded in accordance with the exclusion criteria. A total of 4,313 patients, including 2,631 (61.0\%) male patients, were enrolled in this study, as listed in Table I. Eligible patients were classified into three groups as follows: Group A, 1246 patients $(28.9 \%)$; group B, 1205 patients $(27.9 \%)$; and group C, 1862 patients $(43.2 \%)$ as Fig. 3. The mean ages were $62.4 \pm 18.7,60.6 \pm 20.4$ and $56.1 \pm 19.3$ years in groups A, B and $\mathrm{C}$, respectively. The rates of the high-risk group detected during the baseline colonoscopy were $36.1 \%$ (450 patients) and $37.2 \%$ (448 patients) in groups $\mathrm{A}$ and $\mathrm{B}$, respectively. The average number of lesions diagnosed as neoplastic were 2.2 and 1.5 lesions in groups $\mathrm{A}$ and $\mathrm{B}$, respectively.

Follow-up colonoscopy. Overall, the median follow-up period and the frequency of colonoscopy were 5.1 years and 2.9 times, respectively. There were no significant differences in the follow-up period among the groups. Moreover, the average number of follow-up colonoscopies was 3.5, 3.2 and 2.1 in groups A-C, respectively.
Incidence of ILs. A total of 259 ILs in 233 patients were newly diagnosed during the follow-up colonoscopies. The incidence rates of ILs in each group were as follows: Group A, 8.0\% $(n=100)$; group $B, 8.6 \%(n=104)$; and group $C, 1.6 \%(n=29)$. No significant difference was found between groups $\mathrm{A}$ and $\mathrm{B}$ in the incidence of ILs (Table II). There were 13 (5.0\%), 57 (22.0\%), $59(22.8 \%), 20(7.7 \%), 94(36.3 \%)$, and 18 (6.9\%) ILs located in the cecum, ascending colon, transverse colon, descending colon, sigmoid, and rectum, respectively. Of these ILs, the macroscopic types were $119(45.9 \%)$ polypoid, $112(43.2 \%)$ flat, and 10 (3.8\%) depressed lesions. Histopathologically, 159 $(61.3 \%)$ ILs were adenoma ( $\geq 10 \mathrm{~mm}), 75$ (29.0\%) were high grade dysplasia, $11(4.2 \%)$ were submucosal invasive cancer, and $14(5.4 \%)$ were advanced cancer (Table III).

The cumulative incidence of ILs at 3 and 5 years were $2.6 / 4.3,2.5 / 4.9$, and $0.6 / 1.1 \%$ in groups A-C, respectively (Table IV).

The cumulative incidence rates of the ILs are presented in Fig. 4. No significant difference was found between 
Table I. Characteristics of patients and ILs diagnosed by a follow-up colonoscopy.

\begin{tabular}{lcccc}
\hline Characteristics & Group A & Group B & Group C & Total \\
\hline Patients (no) & 1,246 & 1,205 & 1,862 & 4,313 \\
Male sex [no. (\%)] & $723(58.0)$ & $736(61.1)$ & $1,172(62.9)$ & $2,631(61.0)$ \\
Age (years \pm SD) & $62.4 \pm 18.7$ & $60.6 \pm 20.4$ & $56.1 \pm 19.3$ & $57.2 \pm 19.5$ \\
Risk stratification & & & & - \\
Low risk & $796(63.9 \%)$ & $757(62.8 \%)$ & - & - \\
High risk & $450(36.1 \%)$ & $448(37.2 \%)$ & - & - \\
Number of neoplastic lesions & $2.2(1-9)$ & $1.5(1-7)$ & $5.2 \pm 1.7$ & $5.1 \pm 1.8$ \\
Follow-up period (years \pm SD) & $5.1 \pm 1.8$ & $5.0 \pm 1.9$ & 2.1 & 2.6 \\
Average number of TCS & 3.1 & 2.9 & & \\
\hline
\end{tabular}

TCS, total colonoscopy; SD, standard deviation; ILs, index lesions.

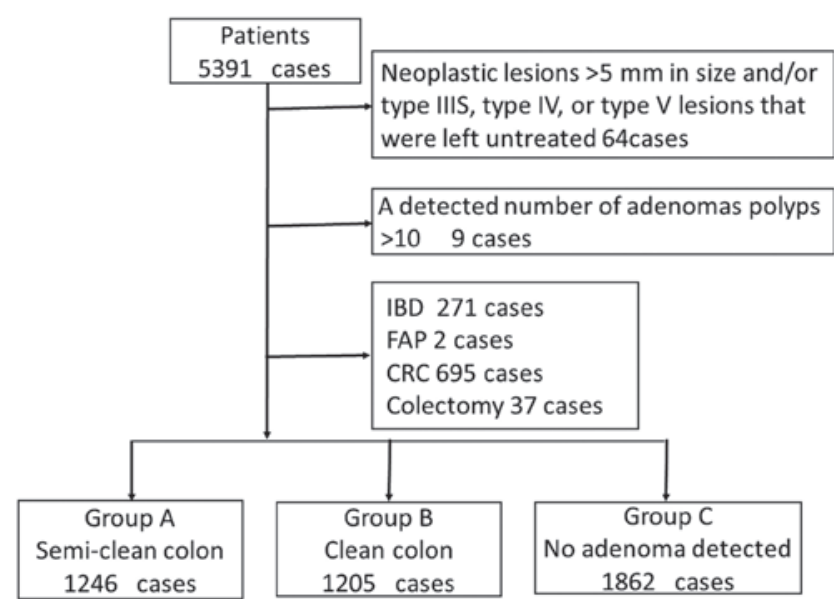

Figure 3. Study chart of this retrospective study.

groups A and B for the cumulative incidence of the ILs. The incidence of ILs in group $C$ was lower than that in both groups $\mathrm{A}$ and $\mathrm{B}(\mathrm{P}<0.01$ and $\mathrm{P}<0.01$, respectively).

In group $\mathrm{B}$, resected DAPs group are significantly higher than group $\mathrm{A}$ in terms of the cumulative incidence rates of the ILs ( $\mathrm{P}=0.012)$ (Fig. 5).

Incidence of invasive and interval cancers. A total of 25 invasive cancers in 23 patients were newly diagnosed during the follow-up colonoscopies (Table II). The incidence rates of invasive cancer in each group were as follows: Group A, $0.6 \%$ $(n=9)$; group $B, 0.6 \%(n=8)$; and group $C, 0.4 \%(n=8)$. The incidence of invasive cancer in Group A was similar to that of group B, as shown in Table II.

In particular, a total of 9 invasive cancers in 9 patients were diagnosed within 36 months following the baseline colonoscopy, as shown in Table II. There was no significant difference in the incidence of invasive cancers diagnosed within 36 months between groups A and B.

The risk factors in future detection of ILs among group $B$. 'The number of neoplastic lesions $\geq 3$ ' and, 'Follow up periods $\geq 5$ years' showed significant high odds rate for future detection of ILs in multivariate logistic regression analyses (Table V).

\section{Discussion}

This study was a retrospective case-control study that analysed the concept of 'semi-clean colon' using Kudo's PIT classification assessed by magnifying the chromoendoscopy for the baseline colonoscopy. The results indicate that there was no significant difference in the cumulative incidence of ILs and invasive cancer among patients with type IIIL PIT DAPs that were left untreated and those in whom all neoplastic lesions were removed. Colonoscopy combined with the removal of all adenomatous polyps has been reported to reduce the risk of recurrent CRC and CRC-related mortality in the National Polyp Study, which has been conducted since $1980(2,3)$. Although the Japanese guidelines indicate that some neoplastic lesions can be left untreated, diminutive polypoid lesions should be followed up (8). To our knowledge, this was the first study to analyse the semi-clean colon strategy and compare it to the clean colon strategy.

In addition, a previous study reported that advanced adenomas typically manifest with measurable interval growth, whereas non-advanced adenomas tend to demonstrate intermediate behaviour. Furthermore, the majority of other benign small polyps tend to remain stable or regress over time (25), and a follow-up of unresected colorectal polyps up to $9 \mathrm{~mm}$ is safe (14). However, some DAPs may have advanced histological features and may even develop into invasive cancer (26). PIT diagnosis by magnifying the chromoendoscopy enables the distinction of neoplasia from non-neoplasia in daily practice. While emerging virtual chromoendoscopy systems [e.g., narrow-band imaging or Fuji intelligent chromoendoscopy $(17,18)]$ are very convenient tools, they do not replace PIT diagnosis in terms of diagnostic ability. Magnifying the chromo-observation is necessary to identify diminutive invasive cancer, even among DAPs. It is essential to perform magnifying chromo-observation to determine the feasibility of leaving diminutive adenomatous lesions in place.

In this study, 39 high grade dysplasia, 5 invasive cancer, 4 advanced cancer were detected in 'semi-clean group'. 
Table II. The incidence of index lesions and invasive cancers.

\begin{tabular}{|c|c|c|c|c|c|c|}
\hline Characteristics & Group A & Group B & P-value ${ }^{a}$ & Group C & P-value & Total \\
\hline Patients, no. & 1,246 & 1,205 & & 1,862 & & 4,313 \\
\hline ILs, no. & 116 & 110 & & 33 & & 259 \\
\hline Patients with ILs, no. (\%) & $100(8.0)$ & $104(8.6)$ & 0.609 & $29(1.6)$ & $<0.01$ & $233(5.4)$ \\
\hline ILs within 36 months, no. & 38 & 36 & & 12 & & 86 \\
\hline Patients with ILs within 36 months, no. (\%) & $32(2.6)$ & $30(2.5)$ & 0.818 & $11(0.6)$ & $<0.01$ & $73(1.7)$ \\
\hline ICs, no. & 9 & 8 & & 8 & & 25 \\
\hline Patient with ICs, no. (\%) & $8(0.6)$ & $7(0.6)$ & 1.000 & $8(0.4)$ & 0.579 & $23(0.5)$ \\
\hline ICs within 36 months, no. & 2 & 3 & & 4 & & 9 \\
\hline Patients with ICs, no. (\%) & $2(0.2)$ & $3(0.2)$ & 1.000 & $4(0.2)$ & 0.721 & $9(0.2)$ \\
\hline
\end{tabular}

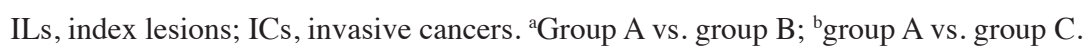

Table III. Clinicopathological characteristics of ILs diagnosed by follow-up colonoscopy.

\begin{tabular}{|c|c|c|c|c|}
\hline Characteristics & Group A & Group B & Group C & Total \\
\hline Number of patients with ILs & 100 & 104 & 29 & 233 \\
\hline Number of ILs & 116 & 110 & 33 & 259 \\
\hline \multicolumn{5}{|l|}{ Location, no. (\%) } \\
\hline Cecum & $4(3.4)$ & $9(8.2)$ & 0 & $13(5.0)$ \\
\hline Ascending & $25(21.6)$ & $23(20.9)$ & $9(27.3)$ & $57(22.0)$ \\
\hline Transverse & $23(19.8)$ & $31(28.2)$ & $5(15.2)$ & $59(22.8)$ \\
\hline Descending & $12(10.3)$ & $8(7.3)$ & 0 & $20(7.7)$ \\
\hline Sigmoid & 47 (41.6) & $32(29.1)$ & $15(45.5)$ & $94(36.3)$ \\
\hline Rectum & $5(4.3)$ & $7(6.4)$ & $6(18.2)$ & $18(6.9)$ \\
\hline \multicolumn{5}{|l|}{ Macroscopic type, no. (\%) } \\
\hline Adenoma and early cancer & $112(96.9)$ & $100(90.6)$ & $29(81.8)$ & $241(93.1)$ \\
\hline Polypoid & $65(56.0)$ & $39(35.5)$ & $15(45.5)$ & $119(45.9)$ \\
\hline Flat & $43(37.1)$ & $58(52.7)$ & $11(33.3)$ & $112(43.2)$ \\
\hline Depressed & $4(3.4)$ & $3(2.7)$ & $3(9.1)$ & $10(3.8)$ \\
\hline \multicolumn{5}{|l|}{ Histopathology, no. (\%) } \\
\hline Adenoma ( $\geq 10 \mathrm{~mm})$ & $68(58.6)$ & $75(68.2)$ & $16(48.5)$ & $159(61.3)$ \\
\hline High grade dysplasia & $39(33.6)$ & $27(24.5)$ & $9(27.3)$ & $75(29.0)$ \\
\hline SM invasive cancer & $5(4.3)$ & $2(1.8)$ & $4(12.1)$ & $11(4.2)$ \\
\hline Advanced cancer & $4(3.4)$ & $6(5.5)$ & $4(12.1)$ & $14(5.4)$ \\
\hline
\end{tabular}

ILs, index lesions; SM, submucosal.

Table IV. The cumulative incidence of index lesions.

\begin{tabular}{|c|c|c|c|c|c|}
\hline \multirow[b]{2}{*}{ Group } & \multicolumn{3}{|c|}{ Cumulative incidence of ILs (\%) } & \multirow[b]{2}{*}{$\mathrm{N}$} & \multirow[b]{2}{*}{ Patients with ILs (N) } \\
\hline & 3-year & 5-year & Maximum follow-up period & & \\
\hline Group A & 2.6 & 4.3 & 8.0 & 1,246 & 100 \\
\hline Group B & 2.5 & 4.9 & 8.6 & 1,205 & 104 \\
\hline Group C & 0.6 & 1.1 & 1.6 & 1,862 & 29 \\
\hline
\end{tabular}

ILs, index lesions; N, number. 
Table V. The risk factors in future detection of index lesions among clean colon group.

\begin{tabular}{lccc}
\hline Factors & OR & $95 \%$ CI & P-value \\
\hline ILs at initial CS & 1.13 & $0.25-5.11$ & 0.86 \\
Neoplastic lesion $\geq 10$ mm at initial CS & 1.89 & $0.45-7.98$ & 0.39 \\
SM invasive cancers at initial CS & 0.39 & $0.05-3.12$ & 0.38 \\
High grade dysplasia at initial CS & 0.87 & $0.42-1.77$ & 0.69 \\
Villous component at initial CS & 1.73 & $0.88-3.42$ & 0.11 \\
The number of neoplastic lesions $\geq 3$ & 2.50 & $1.48-4.20$ & $<.001$ \\
Follow up periods $\geq 5$ years & 3.70 & $2.38-5.88$ & $<0.001$ \\
\hline
\end{tabular}

ILs, index lesions; CS, colonoscopy; SM, submucosal; OR, odds ratio; CI, confidence interval.

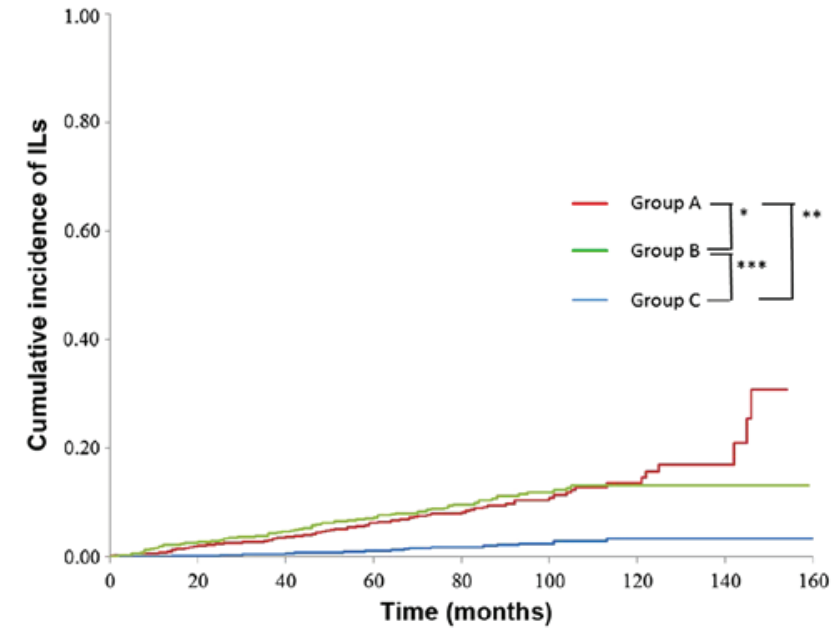

Figure 4. The cumulative incidences of index lesions among groups. ${ }^{*} \mathrm{P}=0.618$, ${ }^{* *} \mathrm{P}<0.01,{ }^{* * *} \mathrm{P}<0.01$

However, Is it not possible to confirm definitely the relationship between untreated DAPs and these cancer lesions.

A previous study reported that the neoplastic lesions found during a colonoscopy are associated with the future risk of incident neoplasms (3), similar to the results of our study. In Japan, Matsuda et al reported that patients with any adenomatous polyps $>6 \mathrm{~mm}$ or intra-mucosal cancer at the time of the initial colonoscopy have a higher risk of advanced lesions than those with no initial neoplasia or small adenomas do (27). In this present study, the clean colon and the semi-clean colon group had a higher incidence of ILs than the internal control group did. This suggested that patients with high-risk polyps were at a future risk of incident neoplasms, regardless of whether their low-risk polyps were removed.

Another issue is that important lesions may be overlooked during the initial colonoscopy but detected during a follow-up colonoscopy. However, in the present study, all examinations were performed under the supervision of experts. It has been reported that one in $13(7.9 \%)$ cases of CRC may be missed on the index colonoscopy (23). In our study, 12 invasive cancers, including eight advanced cancers, were diagnosed with interval cancers within 36 months. Many of these cases were likely because of the presence of lesions that were missed at the time of baseline colonoscopy. In our study, the ascending

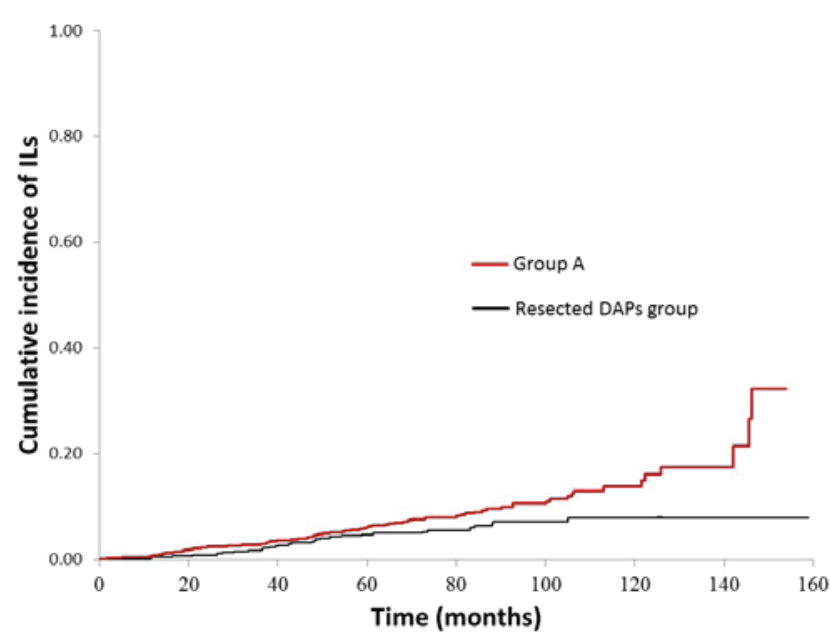

Figure 5. The cumulative incidences of index lesions between groups. $\mathrm{P}=0.012$.

and sigmoid colon were the most common sites of interval cancer. Conversely, a previous report found that a history of prior colonoscopy with polypectomy is one of the strongest risk factors for interval cancer (i.e., early/missed cancer) (23). One reason for this may be that a polypectomy requires more time to perform, and it may be difficult to then set aside an adequate amount of time for observation. One strength of the semi-clean colon strategy is that it may save time.

The 'resect and discard' strategy, as proposed by Ignjatovic et al (28), has the potential to change the standard management of DAPs and reduce the cost of screening and surveillance colonoscopy. The primary benefit of this strategy is the cost savings that can be achieved by reducing the number of polyps that are sent out for histopathological examination, as low-risk lesions are not examined (29). Our strategy for DAPs using PIT classification also has the potential to lower costs by reducing both the number of lesions examined histopathologically, as well as the number of lesions that are treated. The resect and discard strategy for diminutive colorectal lesions was shown to be safe in earlier reports (28). However, the application of this strategy for all diminutive colorectal lesions is unsatisfactory since the evidence from earlier reports was primarily based on sessile lesions (30). 
Recently, the number of patients who receive antiplatelet or anticoagulant drugs has increased. Discontinuation of these medications before the polypectomy increases the risk of certain adverse events (e.g., cerebral vascular disease, myocardial infarction, or even death) $(31,32)$. Heparinization, as an alternative therapy, necessitates hospitalisation, increases cost, and is time-consuming for patients. The present study presents the semi-clean colon strategy for colorectal DAPs as a new treatment option.

Although the cumulative incidence of ILs between the semi-clean and clean colon groups was not significantly different in this study, the Kaplan-Meier curve of the semi-clean colon group exhibited a large increase over the last decade. Thus, we cannot exclude the future potential of DAP to be a malignancy.

However, a surveillance colonoscopy is recommended $<3$ years after endoscopic resection as per the Japanese guidelines (8). In addition to after endosopic resection, the semi-clean colon may be acceptable with a surveillance colonoscopy performed $<3$ years after the initial procedure.

There are several limitations to this study. First, we could not investigate the main indication for the initial colonoscopy. Patients were not limited strictly to asymptomatic cases. However, in this study, outcomes were evaluated based on the findings of the follow-up colonoscopy. Second, this study was a single-centre study. Both expert endoscopists and trainees were involved, but the trainees were always accompanied by experts when determining an on-site PIT diagnosis. Although the validity of the PIT classification was feasible among the expert endoscopists, the diagnostic ability of trainees and their learning curves should be evaluated for a more practical assessment of PIT. Third, this study was a retrospective study and thus might contain some methodological bias, thereby compromising the generalisation of the study results. To compensate for this defect in the study design, we accumulated a relatively large number of samples. However, further multi-centre randomised controlled studies are required to validate these results.

In conclusion, DAPs with type IIIL pit patterns may be left untreated and subsequently observed. The management of colorectal DAPs using pit pattern classification with magnifying chromoendoscopy has the potential to reduce cost, time and risk of polypectomy and repeated colonoscopy. To assess the clinical efficacy of the concept of 'semi-clean colon', further multi-centre prospective clinical trials involving a larger number patients and direct comparisons with a long-term clinical prognosis are required.

\section{Acknowledgements}

The authors thank Enago for editing the manuscript.

\section{References}

1. Morson B: President's address. The polyp-cancer sequence in the large bowel. Proc R Soc Med 67: 451-457, 1974.

2. Zauber AG, Winawer SJ, O'Brien MJ, Lansdorp-Vogelaar I, van Ballegooijen M, Hankey BF, Shi W, Bond JH, Schapiro M, Panish JF, et al: Colonoscopic polypectomy and long-term prevention of colorectal-cancer deaths. N Engl J Med 366: 687-696, 2012.
3. Winawer SJ, Zauber AG, Ho MN, O'Brien MJ, Gottlieb LS, Sternberg SS, Waye JD, Schapiro M, Bond JH, Panish JF, et al: Prevention of colorectal cancer by colonoscopic polypectomy. The national polyp study workgroup. N Engl J Med 329: 1977-1981, 1993.

4. NCCN Clinical Practice Guidelines in Oncology (NCCN Guidelines: Colorectal Cancer Screening. Version 3, 2013.

5. Davila RE, Rajan E, Baron TH, Adler DG, Egan JV, Faigel DO, Gan SI, Hirota WK, Leighton JA, Lichtenstein D, et al: ASGE guideline: Colorectal cancer screening and surveillance. Gastrointest Endosc 63: 546-557, 2006.

6. Lieberman DA, Rex DK, Winawer SJ, Giardiello FM, Johnson DA and Levin TR; United States Multi-Society Task Force on Colorectal Cancer: Guidelines for colonoscopy surveillance after screening and polypectomy: A consensus update by the US multi-society task force on colorectal cancer. Gastroenterology 143: 844-857, 2012.

7. Atkin WS, Valori R, Kuipers EJ, Hoff G, Senore C, Segnan N Jover R, Schmiegel W, Lambert R and Pox C; International Agency for Research on Cancer: European guidelines for quality assurance in colorectal cancer screening and diagnosis. First Edition-Colonoscopic surveillance following adenoma removal. Endoscopy 44 (Suppl 3): SE151-SE163, 2012.

8. Tanaka S, Saitoh Y, Matsuda T, Igarashi M, Matsumoto T, Iwao Y, Suzuki Y, Nishida H, Watanabe T, Sugai T, et al: Evidence-based clinical practice guidelines for management of colorectal polyps. J Gastroenterol 50: 252-260, 2015.

9. Butterly LF, Chaes MP, Pohl H and Fiarman GS: Prevalence of clinically important histology in small adenomas. Clin Gastroenterol Hepatol 4: 343-348, 2006.

10. Rex DK, Overhiser AJ, Chen SC, Cummings OW and Ulbright TM: Estimation of impact of American college of radiology recommendations on CT colonography reporting for resection of high-risk adenoma findings. Am J Gastroenterol 104: 149-153, 2009.

11. Su MY, Ho YP, Chen PC, Chiu CT, Wu CS, Hsu CM and Tung SY: Magnifying endoscopy with indigo carmine contrast for differential diagnosis of neoplastic and nonneoplastic colonic polyps. Dig Dis Sci 49: 1123-1127, 2004.

12. Gupta N, Bansal A, Rao D, Early DS, Jonnalagadda S, Wani SB, Edmundowicz SA, Sharma P and Rastogi A: Prevalence of advanced histological features in diminutive and small colon polyps. Gastrointest Endosc 75: 1022-1030, 2012

13. Matsuda T, Kawano H, Hisabe T, Ikematsu H, Kobayashi N, Mizuno K, Oka S, Takeuchi Y, Tamai N, Uraoka T, et al: Current status and future perspectives of endoscopic diagnosis and treatment of diminutive colorectal polyps. Dig Endosc 26 (Suppl 2): S104-S108, 2014.

14. Kudo S, Tamura S, Nakajima T, Yamano H, Kusaka H and Watanabe $\mathrm{H}$ : Diagnosis of colorectal tumorous lesions by magnifying endoscopy. Gastrointest Endosc 44: 8-14, 1996.

15. Kudo S, Rubio CA, Teixeira CR, Kashida H and Kogure E: Pit pattern in colorectal neoplasia: Endoscopic magnifying view. Endoscopy 33: 367-373, 2001

16. Kobayashi Y, Kudo SE, Miyachi H, Hosoya T, Ikehara N, Ohtsuka K, Kashida H, Hamatani S, Hinotsu S and Kawakami K: Clinical usefulness of pit patterns for detecting colonic lesions requiring surgical treatment. Int J Colorectal Dis 26: 1531-1540, 2011.

17. Wada Y, Kashida H, Kudo SE, Misawa M, Ikehara N and Hamatani S: Diagnostic accuracy of pit pattern and vascular pattern analyses in colorectal lesions. Dig Endosc 22: 192-199, 2010.

18. Sakamoto T, Saito Y, Nakajima T and Matsuda T: Comparison of magnifying chromoendoscopy and narrow-band imaging in estimation of early colorectal cancer invasion depth: A pilot study. Dig Endosc 23: 118-123, 2011.

19. Su MY, Hsu CM, Ho YP, Chen PC, Lin CJ and Chiu CT: Comparative study of conventional colonoscopy, chromoendoscopy, and narrow-band imaging systems in differential diagnosis of neoplastic and nonneoplastic colonic polyps. Am J Gastroenterol 101: 2711-2716, 2006.

20. Huang Q, Fukami N, Kashida H, Takeuchi T, Kogure E, Kurahashi T, Stahl E, Kudo Y, Kimata H and Kudo SE: Interobserver and intra-observer consistency in the endoscopic assessment of colonic pit patterns. Gastrointest Endosc 60: $520-526,2004$

21. The Paris endoscopic classification of superficial neoplastic lesions: Esophagus, stomach, and colon: November 30 to December 1, 2002. Gastrointest Endosc 58 (6 Suppl): S3-S43, 2003. 
22. Hamilton SR and Aaltonen LA (eds): WHO Classification of Tumors: Pathology and Genetics of Tumors of the Digestive System. IARC Press, Lyon, 2000.

23. Singh H, Nugent Z, Demers AA and Bernstein CN: Rate and predictors of early/missed colorectal cancers after colonoscopy in Manitoba: A population-based study. Am J Gastroenterol 105: 2588-2596, 2010.

24. Singh H, Nugent Z, Mahmud SM, Demers AA and Bernstein CN: Predictors of colorectal cancer after negative colonoscopy: A population-based study. Am J Gastroenterol 105: 663-673; quiz 674, 2010.

25. Pickhardt PJ, Kim DH, Pooler BD, Hinshaw JL, Barlow D, Jensen D, Reichelderfer M and Cash BD: Assessment of volumetric growth rates of small colorectal polyps with CT colonography: A longitudinal study of natural history. Lancet Oncol 14: 711-720, 2013.

26. Oka S, Tanaka S, Nakadoi K, Asayama N and Chayama K: Endoscopic features and management of diminutive colorectal submucosal invasive carcinoma. Dig Endosc 26 (Suppl 2): S78-S83, 2014

27. Matsuda T, Fujii T, Sano Y, Kudo S, Oda Y, Igarashi M, Iishi H, Murakami Y, Ishikawa H, Shimoda T, et al: Five-year incidence of advanced neoplasia after initial colonoscopy in Japan: A multicenter retrospective cohort study. Jpn J Clin Oncol 39: 435-442, 2009.
28. Ignjatovic A, East JE, Suzuki N, Vance M, Guenther T and Saunders BP: Optical diagnosis of small colorectal polyps at routine colonoscopy (Detect InSpect ChAracterise Resect and Discard; DISCARD trial): A prospective cohort study. Lancet Oncol 10: 1171-1178, 2009.

29. Rex DK, Kahi C, O'Brien M, Levin TR, Pohl H, Rastogi A, Burgart L, Imperiale T, Ladabaum U, Cohen J and Lieberman DA: The American society for gastrointestinal endoscopy PIVI (preservation and incorporation of valuable endoscopic innovations) on real-time endoscopic assessment of the histology of diminutive colorectal polyps. Gastrointest Endosc 73: 419-422, 2011.

30. Gupta N, Bansal A, Rao D, Early DS, Jonnalagadda S, Edmundowicz SA, Sharma P and Rastogi A: Accuracy of in vivo optical diagnosis of colon polyp histology by narrow-band imaging in predicting colonoscopy surveillance intervals. Gastrointest Endosc 75: 494-502, 2012.

31. Sibon I and Orgogozo JM: Antiplatelet drug discontinuation is a risk factor for ischemic stroke. Neurology 62: 1187-1189, 2004.

32. Blacker DJ, Wijdicks EF and McClelland RL: Stroke risk in anticoagulated patients with atrial fibrillation undergoing endoscopy. Neurology 61: 964-968, 2003. 